\title{
Simulation Study of Adjusted Spatial Weighting Method to Estimate Missing Rainfall Data
}

\author{
Muhammad Az-zuhri Azman*, Roslinazairimah Zakaria and Siti Zanariah Satari
}

Faculty of Industrial Sciences \& Technology, Universiti Malaysia Pahang, Lebuhraya Tun

Razak, 26300, Gambang, Kuantan, Pahang, Malaysia

\begin{abstract}
Missing value especially in environmental study is a common problem including in rainfall modelling. Incomplete data will affect the accuracy and efficiency in any modelling process. In this study, simulation method is used to demonstrate the efficiency of the old normal ratio inverse distance correlation weighting method (ONRIDCWM) in solving missing rainfall data. The simulation study is used to identify the best parameters for correlation power of $p$, percentage of missing value and sample size, $n$ of the ONRIDCWM through simulating for 10,000 times by varying the value of the parameters systematically. The results of the simulation are compared with other available weighting methods. The estimated complete rainfall data of the target station are compared and assessed with the observed data from the neighbouring station using mean, estimated bias (EB) and estimated root mean square error (ERMSE). The results show that ONRIDCWM is better than the other weighting methods for the correlation power of $p$ at least four. For illustration of the weighting method, monthly rainfall data from Pahang is used to demonstrate the efficiency of the method using three error indices: S-Index, mean absolute error (MAE) and correlation, $R$.
\end{abstract}

\section{INTRODUCTION}

Missing values in rainfall data is a common and unavoidable problem faced during statistical analysis. The reason of having missing rainfall data are due to the human error when measuring the rainfall amount, malfunction of the instrument for a certain period of time especially during the extreme rainfall events, unsystematic way when storing the rainfall data or relocation of meteorological rainfall station. Thus, the problem of having missing value required an appropriate method or technique to handle it effectively as it reduces the statistical power of a study which can produce the biased estimates and can have a significant effect on the conclusions that can be drawn from the rainfall data(Ahmad Radi et al., 2015; Azman et al., 2015; Hasana \& Crokea, 2013).

Basically, the procedures to handle missing rainfall values can be divided into three major classes as deterministic, stochastic and artificial intelligence based methods (Campozano et. al., 2014). In this study the deterministic approach is applied. Three advantages of using deterministic approaches are robustness, easy for implementation and computationally efficient (Caldera et. al., 2016; Campozano et. al., 2014; Silva et. al., 2007). The deterministic approach is based on mathematical models which considering certain factors such as distance and correlation for imputing the missing rainfall data. However, the best selection method for estimating missing rainfall values can be varied for different regions depending on their rainfall patterns, spatial and temporal distributions.

In this study, simulation method is used to determine the efficiency of old normal ratio inverse distance correlation weighting method (ONRIDCWM) proposed by Azman et. al., (2015) in completing the missing rainfall data of a selected target station. All the results of ONRIDCWM are compared 
to other weighting method by Suhalia et. al., (2008) using the performance indicator of mean, estimated bias (EB) and estimated root mean square errors (ERMSE).

\section{ESTIMATION WEIGHTING METHOD}

The formulae of weighting methods suggested by Suhalia et al., (2008)(CCWM, CCIDWM, NRIDWM and ONRIDWM) are as follows:

Correlation Coefficient Weighting Method (CCWM)

$$
W_{i}=\frac{\rho_{i t}^{p}}{\sum_{\substack{i=1 \\ i \neq 1}}^{N} \rho_{i t}^{p}}
$$

where $\rho_{i t}^{p}$ is the correlation coefficient between the target station $S_{t}$ and the $i^{\text {th }}$ neighbouring stations with $p=2$.

Correlation Coefficient with Inverse Distance Weighting Method (CCIDWM)

$$
W_{i}=\frac{\rho_{i t}^{p} d_{i t}^{-2}}{\sum_{\substack{i=1 \\ i \neq 1}}^{N} \rho_{i t}^{p} d_{i t}^{-2}}
$$

where $\rho_{i t}^{p}$ is the coefficient of correlation between the target station $t$ and the $i^{\text {th }}$ neighbouring stations with $p=2$ while $d_{i t}$ is the distance between the target station $S_{t}$ and the $i^{\text {th }}$ neighbouring station.

Normal Ratio with Inverse Distance Weighting Method (NRIDWM)

$$
W_{i}=\frac{\left(n_{i}-2\right) \rho_{i t}^{2}\left(1-\rho_{i t}^{2}\right)^{-1} d_{i t}^{-2}}{\sum_{\substack{i=1 \\ i \neq 1}}^{N}\left(n_{i}-2\right) \rho_{i t}^{2}\left(1-\rho_{i t}^{2}\right)^{-1} d_{i t}^{-2}}
$$

where $\rho_{i t}^{2}$ is the square of coefficient of correlation of daily rainfall data between the target station, $S_{t}$ and $i^{\text {th }}$ neighbouring station; $n_{i}$ is the length of data or number of points that are used to compute the correlation coefficient; $d_{i t}$ is the distance between the target station, $S_{t}$ and the $i^{\text {th }}$ neighbouring station and $W_{i}$ is the resultant weight.

Old Normal Ratio with Inverse Distance Weighting Method (ONRIDWM)

$$
W_{i}=\frac{\frac{\mu_{t}}{\mu_{i}} d_{i t}^{-2}}{\sum_{\substack{i=1 \\ i \neq 1}}^{N} \frac{\mu_{t}}{\mu_{i}} d_{i t}^{-2}}
$$

where $d_{i t}$ is the distance between the target station and the $i^{\text {th }}$ neighbouring station while $\mu_{t}$ and $\mu_{i}$ are the sample mean of available data at the target station $S_{t}$ and the $i^{\text {th }}$ neighbouring station respectively and $W_{i}$ is the resultant weight.

\section{A. Modification of Estimation Weighting}

\section{Method}

\section{Old Normal Ratio with Inverse Distance and Correlation Weighting Method} (ONRIDCWM)

The weighting method proposed by Azman et al., (2015) is known as old normal ratio with inverse distance and correlation weighting method (ONRIDCWM) and given by

$$
W_{i}=\frac{\rho_{i t}^{p} \frac{\mu_{t}}{\mu_{i}} d_{i t}^{-2}}{\sum_{\substack{i=1 \\ i \neq 1}}^{N} \rho_{i t}^{p} \frac{\mu_{t}}{\mu_{i}} d_{i t}^{-2}}
$$

where $W_{i}$ is the respective weight, $\rho_{i t}^{p}$ is the correlation coefficient of power $p, \mu_{t}$ and $\mu_{i}$ are the sample mean of available data at the target station $t$ and the $i^{\text {th }}$ neighbouring station respectively and $d_{i t}$ is the distance between $S_{t}$ and the $i^{t h}$ neighbouring station respectively. 


\section{SIMULATION STUDIES}

In this simulation study, program is written using $R$ language. Five different methods considered will be referred as CCWM, CIDWM, NRIDWM, ONRIDWM and ONRIDCWM. In the simulation process, the power of correlation, $p$ for ONRIDCWM formula is varied from $p=1,2,3,4,5$. For each sample, different percentages of missing values (PMV) are assigned: $5 \%, 15 \%$ and $30 \%$, respectively. The estimated rainfall amount $\left(X_{t}\right)$ is calculated using

$$
X_{t}=\sum_{\substack{i=1 \\ i \neq 1}}^{N} W_{i} X_{i}
$$

where $X_{t}$ is the estimated value of the missing data at the target station $S_{t} ; N$ is the number of neighbouring stations; $X_{i}$ is the observation at the $i^{\text {th }}$ neighbouring station and $W_{i}$ is the weight of the $i^{\text {th }}$ neighbouring station with constraint $\sum_{i=1}^{N} W_{i}=1$.

The data is fitted to the gamma model of two parameters, shape ( $\alpha$ ) and scale ( $\beta$ ) and the pdf is given by

$$
\begin{aligned}
& f(x ; \alpha, \beta)=\frac{\beta^{-\alpha} x^{\alpha-1}}{\Gamma(\alpha)} \exp \left(\frac{-x}{\beta}\right), \\
& \alpha>0, \beta>0, x>0 .
\end{aligned}
$$

The simulation process are repeated for 10,000 times, and the values of $X$ are drawn from $X \sim \Gamma(0.7,335)$ with different sample sizes, $n=200,600,1200$. The sets of synthetic data generated are compared and assessed using the performance indicators; mean of the estimated parameters $(\hat{\theta})$, estimated bias (EB) and estimated root mean square errors (ERMSE). The formula for mean of the estimated parameters are given by

$$
\text { Mean of } \hat{\theta}: \overline{\hat{\theta}}=\frac{1}{\operatorname{simu}} \sum \hat{\theta}_{j}
$$

where simu is the number of simulations. So, in this case the value of simu is 1000o. The parameters in the study are $\alpha$ and $\beta$. These values of mean are used to calculate the EB for both parameters of $\alpha$ and $\beta$. The formula of EB is given by

$$
\mathrm{EB} \text { of } \hat{\theta}=|\overline{\hat{\theta}}-\theta|
$$

ERMSE is used to measure the difference between the predicted values by a model and the observed values. The ERMSE formula of the parameters is

$$
\text { ERMSE of } \hat{\theta}=\sqrt{\frac{1}{\operatorname{sim} u} \sum\left(\hat{\theta}_{j}-\theta\right)^{2}}
$$

The smallest value of EB and ERMSE will be chosen and this demonstrate that the estimated parameters are good.

\section{RESULTS AND DISCUSSION}

This study compares the performance of weighting method proposed by Suhaila et al., (2008) and Azman et al., (2015), refer to Table 2 and Table 3. It is observed that, when the power of correlation coefficient is $p=4$ and $p=5$, as the number of sample sizes increasing, the mean of the estimated shape and scale parameters approach the true shape and scale parameters and the error assessment using EB and ERMSE become smaller. In contrast, when the percentage missing values increasing, the mean of the estimated shape and scale parameters diverge from the true shape and scale parameters and the error of EB and ERMSE is increasing. Thus, ONRIDCWM with correlation power of $p=4$ and $p=5$ outperform the other methods for all different percentages of missing values and sample sizes. It is also noted that, ONRIDWM is considered less superior than other methods by Suhaila et al., (2008). However, by including the correlation of power, $p$ the results of ONRIDCWM improved the efficiency when $p=4$ and $p=5$. 


\section{ILLUSTRATIVE EXAMPLES \\ USING RAINFALL DATA FROM PAHANG STATIONS}

In this section, rainfall data from Pahang was selected to show the performance of the proposed method ONRIDCWM by Azman et. al., (2015) and compare with Suhaila et al., (2008) methods. A good performance of weighting method indicates the high value for both values of S-index and correlation $(R)$ but low for mean absolute error (MAE) value.

Table 1. Comparison of estimation method based on S-Index, MAE and $R$ for various percentages of missing rainfall data in Pahang

\begin{tabular}{|c|c|c|c|}
\hline \multirow{2}{*}{ Methods } & $5 \%$ & $15 \%$ & $30 \%$ \\
\hline & \multicolumn{3}{|c|}{ S-Index } \\
\hline CCWM & 0.847 & 0.842 & 0.827 \\
\hline CIDWM & 0.798 & 0.790 & 0.783 \\
\hline NRIDWM & 0.817 & 0.809 & 0.794 \\
\hline ONRIDWM & 0.808 & 0.803 & 0.786 \\
\hline \multirow[t]{2}{*}{ ONRIDCWM } & 0.856 & 0.845 & 0.831 \\
\hline & \multicolumn{3}{|c|}{ MAE } \\
\hline CCWM & 85.519 & 85.820 & 86.057 \\
\hline CIDWM & 85.969 & 85.989 & 86.474 \\
\hline NRIDWM & 85.819 & 85.910 & 86.309 \\
\hline ONRIDWM & 85.889 & 85.938 & 86.391 \\
\hline \multirow[t]{2}{*}{ ONRIDCWM } & 85.447 & 85.798 & 85.882 \\
\hline & \multicolumn{3}{|c|}{$R$} \\
\hline CCWM & 0.785 & 0.763 & 0.753 \\
\hline CIDWM & 0.756 & 0.703 & 0.658 \\
\hline NRIDWM & 0.769 & 0.730 & 0.688 \\
\hline ONRIDWM & 0.763 & 0.724 & 0.673 \\
\hline ONRIDCWM & 0.788 & 0.778 & 0.769 \\
\hline
\end{tabular}

In this study, it is observed that from

Table 1, ONRIDCWM using the correlation power of $p=4$ give the best results as compared to other methods from Suhaila et al., (2008). However, when the number of sample sizes is increasing, the performance of each estimation method tends to decrease slightly in S-index and
$R$ but to increase slightly for MAE. Thus, this results shows that the proposed weighting method by Azman et al., (2015) able to improve the existing weighting methods.

\section{CONCLUSION}

In this study, simulation method is used to demonstrate the efficiency of the old normal ratio inverse distance correlation weighting method (ONRIDCWM) in solving missing rainfall data. The ONRIDCWM formula includes the correlation coefficient of power $p$ whereas the old normal ratio inverse distance (ONRIDWM) formula suggested by Suhaila et. al., (2008) did not include the correlation coefficient of power $p$ term. Analysis using simulation study found that the ONRIDCWM formula is able to provide optimal result when the power of correlation is four and five with minimum errors. Hence, ONRIDCWM improved the results of ONRIDWM by Suhaila et. al., (2008). The results also show that ONRIDCWM is better than the other weighting methods for the correlation power of $p$ at least four. For future study, the viability of the suggested method ONRIDCWM can be tested using other variations of factors including missing values more than $30 \%$.

\section{ACKNOWLEDGEMENTS}

The authors would like to convey thanks to Universiti Malaysia Pahang for the financial support (RDU1703208). We thank the anonymous referees for their useful suggestions. 


\section{REFERENCES}

Ahmad Radi, N. F., Zakaria, R., \& Azman, M. A. 2015, Estimation of Missing Rainfall Data Using Spatial Interpolation and Imputation Methods. AIP Conference Proceedings, 1643, 42-48.

Azman, M. A., Zakaria, R., \& Ahmad Radi, N. F. 2015, Estimation of Missing Rainfall Data in Pahang Using Modified Spatial Interpolation Weighting Methods. AIP Conference Proceedings, 1643, 65-72.

Caldera, H. P. G. M., Piyathisse, V. R. P. C., \& Nandalal, K. D. W. 2016, A Comparison of Methods of Estimating Missing Daily Rainfall Data. Engineer: Journal of the Institution of Engineers, 49(4), 1-8.

Campozano, L., Sánchez, E., Aviles, A., \& Samaniego, E. 2014, Evaluation of Infilling Methods for Time Series of Daily Precipitation and Temperature: The case of the Ecuadorian Andes. Maskana, 5(1), 99-115.

Hasana, M., \& Crokea, B. 2013, Filling Gaps in Daily Rainfall Data: A Statistical Approach. 2oth International Congress on Modelling and Simulation, Adelaide, Australia, (December), 1-6.

Silva, R. P. De, Dayawansa, N. D. K., \& Ratnasiri, M. D. 2007, A Comparison of Methods Used in Estimating Missing Rainfall Data, (May), 101-108.

Suhalia, J., Sayang, M. D., \& Jemain, A. A. 2008, Revised Spatial Weighting Methods for Estimation of Missing Rainfall Data. Asia-Pacific Journal of Atmospheric Sciences, 44(2), 93-104. 
Table 2. Simulation results of performance indicator for mean of estimated shape parameter, $\overline{\hat{\alpha}}$ and error assessment (EB and ERMSE) with different power of correlations, number of sample sizes and percentages of missing value (

$$
\alpha=0.7 \text { ) }
$$

\begin{tabular}{|c|c|c|c|c|c|c|c|c|c|c|c|}
\hline \multirow[t]{2}{*}{$\begin{array}{c}\text { Performance } \\
\text { Indicator }\end{array}$} & \multirow{2}{*}{\multicolumn{2}{|c|}{$\begin{array}{c}\text { No. of Sample } \\
\text { Size }\end{array}$}} & \multicolumn{3}{|c|}{200} & \multicolumn{3}{|c|}{600} & \multicolumn{3}{|c|}{1200} \\
\hline & & & 5 & 15 & 30 & 5 & 15 & 30 & 5 & 15 & 30 \\
\hline \multirow{9}{*}{$\begin{array}{c}\text { Mean } \\
\text { of } \hat{\alpha}\end{array}$} & \multicolumn{2}{|l|}{ CCWM } & 0.713 & 0.723 & 0.742 & 0.707 & 0.718 & 0.737 & 0.706 & 0.716 & 0.735 \\
\hline & \multicolumn{2}{|l|}{ CIDWM } & 0.719 & 0.743 & 0.785 & 0.713 & 0.739 & 0.779 & 0.712 & 0.737 & 0.777 \\
\hline & \multicolumn{2}{|l|}{ NRIDWM } & 0.717 & 0.736 & 0.769 & 0.711 & 0.731 & 0.764 & 0.710 & 0.729 & 0.764 \\
\hline & \multicolumn{2}{|l|}{ ONRIDWM } & 0.718 & 0.740 & 0.779 & 0.712 & 0.736 & 0.771 & 0.712 & 0.734 & 0.771 \\
\hline & \multirow{5}{*}{$\begin{array}{c}p-\text { power of } \\
\text { ONRIDCWM }\end{array}$} & 1 & 0.717 & 0.736 & 0.770 & 0.711 & 0.731 & 0.764 & 0.710 & 0.729 & 0.764 \\
\hline & & 2 & 0.715 & 0.729 & 0.756 & 0.709 & 0.724 & 0.751 & 0.708 & 0.723 & 0.750 \\
\hline & & 3 & 0.714 & 0.725 & 0.746 & 0.707 & 0.719 & 0.740 & 0.707 & 0.718 & 0.739 \\
\hline & & 4 & 0.712 & 0.721 & $\mathbf{0 . 7 3 7}$ & 0.706 & 0.715 & 0.732 & 0.705 & 0.714 & 0.730 \\
\hline & & 5 & 0.711 & 0.717 & 0.731 & 0.705 & 0.712 & 0.726 & 0.704 & 0.711 & 0.724 \\
\hline \multirow{9}{*}{ EB } & \multicolumn{2}{|l|}{ CCWM } & 0.013 & 0.023 & 0.042 & 0.007 & 0.018 & 0.037 & 0.006 & 0.016 & 0.035 \\
\hline & \multicolumn{2}{|l|}{ CIDWM } & 0.019 & 0.043 & 0.085 & 0.013 & 0.039 & 0.079 & 0.012 & 0.037 & 0.077 \\
\hline & \multicolumn{2}{|l|}{ NRIDWM } & 0.017 & 0.036 & 0.069 & 0.011 & 0.031 & 0.064 & 0.010 & 0.029 & 0.064 \\
\hline & \multicolumn{2}{|l|}{ ONRIDWM } & 0.018 & 0.040 & 0.079 & 0.012 & 0.036 & 0.071 & 0.012 & 0.034 & 0.071 \\
\hline & \multirow{5}{*}{$\begin{array}{c}p-\text { power of } \\
\text { ONRIDCWM }\end{array}$} & 1 & 0.017 & 0.036 & 0.070 & 0.011 & 0.031 & 0.064 & 0.010 & 0.029 & 0.064 \\
\hline & & 2 & 0.015 & 0.029 & 0.056 & 0.009 & 0.024 & 0.051 & 0.008 & 0.023 & 0.050 \\
\hline & & 3 & 0.014 & 0.025 & 0.046 & 0.007 & 0.019 & 0.040 & 0.007 & 0.018 & 0.039 \\
\hline & & 4 & 0.012 & 0.021 & $\mathbf{0 . 0 3 7}$ & 0.006 & 0.015 & 0.032 & 0.005 & 0.014 & 0.030 \\
\hline & & 5 & 0.011 & 0.017 & $\mathbf{0 . 0 3 1}$ & O.005 & 0.012 & 0.026 & 0.004 & 0.011 & 0.024 \\
\hline \multirow{9}{*}{ ERMSE } & \multicolumn{2}{|l|}{ CCWM } & 0.064 & 0.069 & 0.088 & 0.037 & 0.044 & 0.068 & 0.027 & 0.037 & 0.063 \\
\hline & \multicolumn{2}{|l|}{ CIDWM } & 0.066 & 0.078 & 0.113 & 0.039 & 0.055 & 0.093 & 0.029 & 0.048 & 0.088 \\
\hline & \multicolumn{2}{|l|}{ NRIDWM } & 0.065 & 0.076 & 0.104 & 0.038 & 0.052 & 0.084 & 0.028 & 0.043 & 0.080 \\
\hline & \multicolumn{2}{|l|}{ ONRIDWM } & 0.065 & 0.078 & 0.111 & 0.038 & 0.054 & 0.090 & 0.028 & 0.046 & 0.085 \\
\hline & \multirow{5}{*}{$\begin{array}{r}p-\text { power of } \\
\text { ONRIDCWM }\end{array}$} & 1 & 0.065 & 0.076 & 0.104 & 0.038 & 0.052 & 0.084 & 0.028 & 0.043 & 0.080 \\
\hline & & 2 & 0.064 & 0.073 & 0.096 & 0.037 & 0.048 & 0.076 & 0.027 & 0.040 & 0.071 \\
\hline & & 3 & 0.064 & 0.070 & 0.090 & 0.037 & 0.046 & 0.069 & 0.027 & 0.037 & 0.065 \\
\hline & & 4 & 0.064 & 0.069 & 0.086 & $\mathbf{0 . 0 3 7}$ & 0.044 & 0.065 & 0.026 & $\mathbf{0 . 0 3 5}$ & 0.059 \\
\hline & & 5 & 0.063 & 0.067 & 0.083 & 0.036 & 0.043 & 0.061 & 0.026 & 0.034 & 0.055 \\
\hline
\end{tabular}


Table 3. Simulation results of performance indicator for mean of estimated scale parameter, $\overline{\hat{\beta}}$ and error assessment (EB and ERMSE) with different power of correlations, number of sample sizes and percentages of missing value ( $\beta=355.0$ )

\begin{tabular}{|c|c|c|c|c|c|c|c|c|c|c|c|}
\hline \multirow{2}{*}{$\begin{array}{l}\text { Performance } \\
\text { Indicator }\end{array}$} & \multirow{2}{*}{\multicolumn{2}{|c|}{\begin{tabular}{|c|} 
No. of Sample Size \\
Percentage of \\
missing values (\%)
\end{tabular}}} & \multicolumn{3}{|c|}{200} & \multicolumn{3}{|c|}{600} & \multicolumn{3}{|c|}{1200} \\
\hline & & & 5 & 15 & 30 & 5 & 15 & 30 & 5 & 15 & 30 \\
\hline \multirow{9}{*}{ Mean of $\hat{\beta}$} & \multicolumn{2}{|l|}{ CCWM } & 330.295 & 323.717 & 311.312 & 330.856 & 323.598 & 312.876 & 329.910 & 325.241 & 312.800 \\
\hline & \multicolumn{2}{|l|}{ CIDWM } & 327.518 & 315.305 & 294.266 & 327.814 & 314.517 & 295.828 & 327.114 & 315.648 & 295.921 \\
\hline & \multicolumn{2}{|l|}{ NRIDWM } & 328.390 & 318.168 & 300.838 & 328.936 & 317.780 & 301.743 & 328.091 & 318.617 & 301.753 \\
\hline & \multicolumn{2}{|c|}{ ONRIDWM } & 327.752 & 316.335 & 296.867 & 328.341 & 315.990 & \begin{tabular}{|l|}
298.237 \\
\end{tabular} & 327.586 & 316.428 & 298.829 \\
\hline & \multirow{5}{*}{$\begin{array}{c}p \text { - power of } \\
\text { ONRIDCWM }\end{array}$} & 1 & 328.350 & 318.042 & 300.610 & 328.919 & 317.739 & 301.688 & 328.079 & 318.590 & 301.714 \\
\hline & & 2 & 329.213 & 320.616 & 305.690 & 329.851 & 320.535 & 307.241 & 328.970 & 321.536 & 307.036 \\
\hline & & 3 & 329.913 & 322.941 & 310.012 & 330.654 & 322.864 & 311.800 & 329.694 & 323.965 & 311.563 \\
\hline & & 4 & 330.469 & 324.777 & 313.420 & 331.275 & 324.618 & 315.282 & 330.257 & 325.827 & 315.062 \\
\hline & & 5 & 330.903 & 326.210 & 316.035 & 331.747 & 325.965 & $317 \cdot 913$ & 330.700 & 327.250 & 317.789 \\
\hline \multirow{9}{*}{ EB } & \multicolumn{2}{|l|}{ CCWM } & 4.705 & 11.283 & 23.688 & 4.144 & 11.402 & 22.124 & 5.090 & 9.759 & 22.200 \\
\hline & \multicolumn{2}{|l|}{ CIDWM } & 7.482 & 19.695 & 40.734 & 7.186 & 20.483 & 39.172 & 7.886 & $19 \cdot 352$ & 39.079 \\
\hline & \multicolumn{2}{|l|}{ NRIDWM } & 6.610 & 16.832 & 34.162 & 6.064 & 17.220 & 33.257 & 6.909 & 16.383 & 33.247 \\
\hline & \multicolumn{2}{|l|}{ ONRIDWM } & 7.248 & 18.665 & 38.133 & 6.659 & 19.010 & 36.763 & 7.414 & 18.572 & 36.171 \\
\hline & \multirow{5}{*}{$\begin{array}{c}p \text { - power of } \\
\text { ONRIDCWM }\end{array}$} & 1 & 6.650 & 16.958 & 34.390 & 6.081 & 17.261 & $33 \cdot 312$ & 6.921 & 16.410 & 33.286 \\
\hline & & 2 & $5 \cdot 787$ & 14.384 & 29.310 & 5.149 & 14.465 & 27.759 & 6.030 & 13.464 & 27.964 \\
\hline & & 3 & 5.087 & 12.059 & 24.988 & $4 \cdot 346$ & 12.136 & 23.200 & $5 \cdot 306$ & 11.035 & 23.437 \\
\hline & & 4 & 4.531 & 10.223 & 21.580 & $3 \cdot 725$ & 10.382 & 19.718 & 4.743 & 9.173 & 19.938 \\
\hline & & 5 & 4.097 & 8.790 & 18.965 & 3.253 & 9.035 & 17.087 & 4.300 & $7 \cdot 750$ & 17.211 \\
\hline \multirow{9}{*}{ ERMSE } & \multicolumn{2}{|l|}{ CCWM } & 40.668 & 41.723 & 49.551 & 23.681 & 27.130 & 35.898 & 17.276 & 20.174 & 32.272 \\
\hline & \multicolumn{2}{|l|}{ CIDWM } & 40.850 & 43.219 & 56.456 & 24.077 & 30.713 & $45 \cdot 956$ & 18.053 & 25.232 & 43.186 \\
\hline & \multicolumn{2}{|l|}{ NRIDWM } & 40.736 & 42.847 & 53.078 & 23.782 & 29.697 & 42.503 & 17.751 & 23.702 & 39.463 \\
\hline & \multicolumn{2}{|l|}{ ONRIDWM } & 40.760 & 43.122 & 54.805 & 23.907 & 30.531 & 45.099 & 17.928 & 24.869 & 41.688 \\
\hline & \multirow{5}{*}{$\begin{array}{c}p \text { - power of } \\
\text { ONRIDCWM }\end{array}$} & 1 & 40.748 & 42.847 & 53.211 & 23.779 & 29.717 & 42.527 & 17.753 & 23.717 & 39.484 \\
\hline & & 2 & 40.709 & 42.325 & 51.057 & 23.646 & 28.425 & 39.163 & 17.500 & 22.173 & 35.806 \\
\hline & & 3 & 40.712 & 41.964 & 49.441 & 23.604 & 27.434 & 36.524 & $17 \cdot 313$ & 20.959 & 32.882 \\
\hline & & 4 & 40.717 & 41.684 & 48.360 & 23.584 & $\mathbf{2 6 . 7 8 7}$ & 34.474 & 17.160 & 20.111 & 30.630 \\
\hline & & 5 & 40.711 & 41.477 & 47.581 & 23.589 & 26.304 & 32.948 & 17.045 & 19.478 & $\mathbf{2 8 . 8 7 5}$ \\
\hline
\end{tabular}

